\title{
Synchronous Melanoma and Pancreas Malignancies Leading to a Discovery of a CDKN2A Mutation in a Patient with No Known Family History
}

\author{
Mary O'Reilly Fergus Keane Ray Mc Dermott \\ St Vincent's University Hospital, Dublin, Ireland
}

\author{
Keywords \\ Synchronous primary malignancies · Pancreatic cancer · Malignant melanoma - Genetic \\ predisposition · CDKN2A mutation
}

\begin{abstract}
We report a case of a 60 -year-old male with metachronous primary malignancies, pancreatic cancer and malignant melanoma which recurred simultaneously. Both cancers were challenging to diagnose and throughout the case at different times, the presence of two active malignancies obscured the clinical picture. A bleeding gastric lesion found in the stomach 6 months after a distal pancreatectomy for pancreatic adenocarcinoma revealed metastatic melanoma, presumed secondary from a melanoma excised from the patient's back 2 years previously. During surgery intended to resect the gastric lesion, peritoneal nodularity was identified, with histology confirming metastatic pancreas cancer. This case highlights two main points of interest. Firstly it emphasises the role for consideration of a genetic predisposition in young patients with more than one primary malignancy. The man in this case was not informed of his family history as he was adopted. If he had knowledge of previous family history, he may have been able to provide information to expedite arrival at the diagnosis of a CDKN2A mutation (melanoma-pancreatic carcinoma syndrome). In addition, this case also raises the issue of the challenges we face when treating synchronous primary malignancies. The two malignancies here behaved equally aggressively and posed obstacles for treatment as there is no mutual method of carcinogenesis that could be targeted with treatment; therefore, treatment modalities had to be chosen to treat each malignancy separately. To date, studies evaluating the role for targeted therapy in the setting of CDKN2A mutations have not conclusively provided meaningful benefits to patients.




\section{Introduction}

Many hereditary predisposition cancer syndromes exist. Hereditary breast and ovarian cancer syndrome, Lynch syndrome (formerly known as hereditary non-polyposis colon cancer) and Li-Fraumeni syndrome are among the most common, but a large number of other less common syndromes exist. Early detection of a cancer syndrome in a patient is crucial for a number of reasons. Firstly, to improve outcomes by allowing prompt diagnosis of a malignancy by screening. Secondly, it allows for more accurate prognostication and knowledge of the trajectory of the diseases associated, and thirdly by allowing for the provision of genetic counselling for relatives that may also be affected by the syndrome.

Melanoma-pancreatic carcinoma syndrome is a rare inherited cancer predisposition syndrome caused by a heterozygous mutation in the CDKN2A gene on chromosome 9p21. Germline CDKN2A mutations are rare in the whole population $(<0.1 \%)$ with clusters seen in familial melanoma probands [1].

The function of CDK2A gene is that it codes for a number of proteins; among them tumour suppressors p16(INK4A) and unrelated p14(ARF) proteins. Specific binding of the p16INK4a protein to CDK4 or CDK6 causes a change in structure in these proteins. This inhibits complex formation between CDK4/6 and cyclin D which has a knock on effect to maintain the retinoblastoma protein in its hypo-phosphorylated and growth-suppressive state, causing cell cycle arrest.

Mutation carriers within families may develop either or both types of cancer [2]. It is inherited in an autosomal dominant manner [3]. Patients with this mutation have a 65-fold increased risk of developing melanoma [4] and a 38-fold increased risk of developing pancreatic cancer compared with the general population [5].

\section{Case Report}

This is the case of a 60-year-old Caucasian male who was known to the Oncology service as he was undergoing six monthly surveillance computed tomography (CT) of the thorax, abdomen and pelvis after diagnosis of malignant melanoma which was excised from his back in 2018. At the time of diagnosis, it was a Stage IIIb lesion with 3/30 nodes positive. Adjuvant radiation was performed to the axilla at the time, but the patient was not deemed suitable for adjuvant immunotherapy because of a past medical history which was significant for dilated cardiomyopathy after previous viral myocarditis. He had an ejection fraction of $50 \%$, atrial fibrillation and previous hepatitis also presumed to be viral.

His son had a melanoma removed at age 22 but given the fact he was adopted, he had no other family history to report. He was an ex-smoker of 20 years with a 30 pack-year history and drank 8 units of alcohol/week. He had a ECOG of 1 and was still active and in full time employment.

In November 2019, the patient was referred to our tertiary centre for work-up of a suspected pancreatic cancer as his CT showed an irregularity in the tail of the pancreas. An endoscopic ultrasound was done to obtain tissue which returned as pancreatic adenocarcinoma. The patient's case was discussed at the Hepatobiliary Multidisciplinary Team meeting, and it was decided that a Positron Emission Technology (PET) scan should be performed given the previous diagnosis of melanoma. The PET scan showed a 2.1-cm mass with a SUV max of 5.64 at junction of pancreatic body and tail, but no other significant uptake was seen. The decision was made for surgery. Given a significant amount of inflammation around the pancreas during laparotomy, the surgeons performed a distal pancreatectomy, splenectomy, and partial colectomy. Histology revealed a pT3N1 pancreatic adenocarcinoma with clear

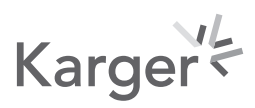


resection margins (R0), no lymphovascular invasion and 2/50 nodes positive. Consideration was given to commencing the patient on adjuvant FOLFIRINOX; however, given his medical comorbidities, a decision was taken to commence with adjuvant FOLFOX and escalate to FOLFIRINOX if tolerated.

In May 2020, as the patient was approaching his 5th cycle of FOLFOX, he was admitted with an upper GI bleed. OGD revealed an abnormal gastric mass which was presumed to be a pancreatic metastasis. However, histologically the tumour showed diffuse positivity for the melanocytic marker melan A, and a diagnosis of malignant melanoma in the stomach was made. Limited melanoma genomic panel showed that the melanoma was BRAF mutated.

He had a PET scan with a view to surgery. The PET showed radiotracer uptake only in gastric mucosa, so a decision to proceed to laparotomy and subtotal gastrectomy was made. Unfortunately, at laparotomy multiple peritoneal nodules were seen, so the procedure was abandoned and peritoneal biopsy performed. The peritoneal biopsy showed adenocarcinoma, with immunohistochemistry consistent with pancreatic origin. The patient received then palliative radiotherapy to prevent further gastrointestinal bleeding.

The pancreatic tissue was sent for next-generation sequence analysis by Foundation One Test to ascertain as to whether there were any actionable mutations to guide treatment. The genetic sequencing demonstrated a CDK2NA/B mutation. A putative diagnosis of melanomapancreatic cancer syndrome was established to be confirmed by germline testing.

In June 2020, the patient presented to Hospital with vomiting and headaches. Magnetic resonance imaging of the brain demonstrated three enhancing lesions reflecting metastatic deposits. They had a haemorrhagic appearance and following multidisciplinary team discussion they were deemed to be consistent with metastatic melanoma. While the patient was undergoing whole brain radiotherapy for the brain metastasis, he was admitted for investigation of constipation and vomiting and was found to have small bowel obstruction secondary to diffuse peritoneal metastases. He passed away in August 2020.

\section{Discussion}

Cancer predisposition syndromes such as this can be very challenging and are often a therapeutic dilemma. In this case, there were two aggressive malignancies, both requiring active treatment. The plan once both diagnoses were made was to treat the patient with a fluorouracil-based regimen to harness the pancreatic lesion first and then to treat the melanoma with a BRAF/MEK inhibitor to gain control of the melanoma.

As we continue to achieve better outcomes for cancer patients as a result of earlier diagnosis and evolving methods of treating malignant tumours, the incidence of patients with more than one primary tumour is increasing [6]. This scenario shows that the clinician should always question the presence of an underlying cancer predisposition syndrome in a young patient with more than one primary malignancy. The fact that the patient was adopted obscured the clinical picture from the outset as he had no previous family history; besides, his son who was diagnosed with melanoma at 22 years of age, but genetic testing was not obtained at that point. Had he known his family history, arriving at a familial cancer predisposition syndrome might have been done sooner.

There is no specific targeted therapy for CDK2NA mutations. It has been hypothesised given the success CDK4/6 inhibitors in the field of human epidermal growth factor receptor 2 (HER 2)-negative metastatic breast cancer [7] that they might show promise in a disease that is thought to be mediated by overactivity of the CDK4/6 proteins. Unfortunately, there have been no meaningful data from early studies investigating CDK4/6 inhibition in patients

\section{Karger' ${ }^{\prime}=$}


with CDK2NA mutations. Undoubtedly, further trials examining treatments to target this mutation and the proteins which are activated when this gene is mutated are needed.

The patient's diagnosis of melanoma-pancreatic syndrome has implications for his children. CDK2NA is a germline mutation inherited in an autosomal dominant manner with variable penetrance [3]. The CDK2NA mutation carries with it a 38-fold increase of developing pancreatic cancer [8] and a 65-fold increase in developing melanoma [4] compared to the general population. The screening recommendations for early detection of melanoma for those with a known mutation are three to six monthly dermatology skin examination as well as education on sun protection and importance of cessation of tobacco use. There is no agreement in the literature on the best practice of screening in pancreatic cancer [9]. This is an area which needs further research and development so should be discussed and assess on an individual basis [9]. The children are of consenting age and have been referred appropriately for genetic testing.

The patient's prognosis here was guarded in light of the two malignancies and their related complications. We attempted to treat his cancer in a sequential manner; addressing the cancer which was most active while attempting to maintain a decent quality of life for the patient; however, complications from both cancers rendered that impossible.

\section{Statement of Ethics}

Written informed consent was obtained from the patient for publication of this case report.

\section{Conflict of Interest Statement}

The authors have no conflict of interest to declare.

\section{Funding Sources}

No funding Sources to declare.

\section{Author Contributions}

Dr. Mary O'Reilly - Writing the case report. Dr. Fergus Keane - Writing and editing the case report. Prof. Ray Mc Dermott - Editing the case report.

\section{References}

1 Helgadottir H, Ghiorzo P, van Doorn R, Puig S, Levin M, Kefford R, et al. Efficacy of novel immunotherapy regimens in patients with metastatic melanoma with germline CDKN2A mutations. J Med Genet. 2020 May; 57(5):316-321.

2 Harinck F, Kluijt I, van der Stoep N, Oldenburg RA, Wagner A, Aalfs CM, et al. Indication for CDKN2A-mutation analysis in familial pancreatic cancer families without melanomas. J Med Genet. 2012;49(6):362 .

3 Oldenburg A, Gabree MJ, Tsao H. Melanoma susceptibility genes and risk assessment. Methods Mol Biol. 2014; 1102:381.

4 H Helgadottir, V Höiom, R Tuominen, K Nielsen, G Jönsson, H Olsson, et al. Germline CDKN2A Mutation Status and Survival in Familial Melanoma Cases. J Natl Cancer Inst. November 2016;108(11):djw135

\section{Karger's}


5 Lynch HT, Fusaro RM, Lynch JF, Brand R. Pancreatic cancer and the FAMMM syndrome. Fam Cancer. 2008; $7(1): 103-12$.

6 Wood ME, Vogel V, Ng A, Foxhall L, Goodwin P, Travis LB. Second malignant neoplasms: assessment and strategies for risk reduction. J Clin Oncol. 2012 Oct 20;30(30):3734-45.

7 Slamon DJ, Neven P, Chia S, Fasching PA, De Laurentiis M, Im SA, et al. Phase III Randomized Study of Ribociclib and Fulvestrant in Hormone Receptor-Positive, Human Epidermal Growth Factor Receptor 2-Negative Advanced Breast Cancer: MONALEESA-3. J Clin Oncol. 2018 Aug 20;36(24):2465-2472.

8 Soura E, Eliades PJ, Shannon K, Stratigos AJ, Tsao H. Hereditary melanoma: Update on syndromes and management: Genetics of familial atypical multiple mole melanoma syndrome. J Am Acad Dermatol. 2016; 74(3):395-10.

9 McWilliams RR, Wieben ED, Rabe KG, Pedersen KS, Wu Y, Sicotte H, et al. Prevalence of CDKN2A mutations in pancreatic cancer patients: implications for genetic counseling. Eur J Hum Genet. 2011;19(4):472-8. 